\title{
THE DENSITY OF PRIMES DIVIDING A TERM IN THE SOMOS-5 SEQUENCE
}

\author{
BRYANT DAVIS, REBECCA KOTSONIS, AND JEREMY ROUSE
}

(Communicated by Matthew A. Papanikolas)

\begin{abstract}
The Somos- 5 sequence is defined by $a_{0}=a_{1}=a_{2}=a_{3}=a_{4}=1$ and $a_{m}=\frac{a_{m-1} a_{m-4}+a_{m-2} a_{m-3}}{a_{m-5}}$ for $m \geq 5$. We relate the arithmetic of the Somos-5 sequence to the elliptic curve $E: y^{2}+x y=x^{3}+x^{2}-2 x$ and use properties of Galois representations attached to $E$ to prove the density of primes $p$ dividing some term in the Somos- 5 sequence is equal to $\frac{5087}{10752}$.
\end{abstract}

\section{INTRODUCTION AND STATEMENT OF RESUlTS}

There are many results in number theory that relate to a determination of the primes dividing some particular sequence. For example, it is well known that if $p$ is a prime number, then $p$ divides some term of the Fibonacci sequence, defined by $F_{0}=0, F_{1}=1$, and $F_{n}=F_{n-1}+F_{n-2}$ for $n \geq 2$. Students in elementary number theory learn that a prime $p$ divides a number of the form $n^{2}+1$ if and only if $p=2$ or $p \equiv 1(\bmod 4)$.

In 1966, Hasse proved in [4] that if $\pi_{\text {even }}(x)$ is the number of primes $p \leq x$ so that $p \mid 2^{n}+1$ for some $n$, then

$$
\lim _{x \rightarrow \infty} \frac{\pi_{\text {even }}(x)}{\pi(x)}=\frac{17}{24} .
$$

Note that a prime number $p$ divides $2^{n}+1$ if and only if 2 has even order in $\mathbb{F}_{p}^{\times}$.

A related result is the following. The Lucas numbers are defined by $L_{0}=2$, $L_{1}=1$ and $L_{n}=L_{n-1}+L_{n-2}$ for $n \geq 2$. In 1985, Lagarias proved (see [9] and [10]) that the density of primes dividing some Lucas number is $2 / 3$. Given a prime number $p$, let $Z(p)$ be the smallest integer $m$ so that $p \mid F_{m}$. A prime $p$ divides $L_{n}$ for some $n$ if and only if $Z(p)$ is even. In [2, Paul Cubre and the third author prove a conjecture of Bruckman and Anderson on the density of primes $p$ for which $m \mid Z(p)$, for an arbitrary positive integer $m$.

In the early 1980s, Michael Somos discovered integer-valued non-linear recurrence sequences. The Somos- $k$ sequence is defined by $c_{0}=c_{1}=\cdots=c_{k-1}=1$ and

$$
c_{m}=\frac{c_{m-1} c_{m-(k-1)}+c_{m-2} c_{m-(k-2)}+\cdots+c_{m-\left\lfloor\frac{k}{2}\right\rfloor} c_{m-\left\lceil\frac{k}{2}\right\rceil}}{c_{m-k}}
$$

for $m \geq k$. Despite the fact that division is involved in the definition of the Somos sequences, the values $c_{m}$ are integral for $4 \leq k \leq 7$. Fomin and Zelevinsky [3] show that the introduction of parameters into the recurrence results in the $c_{m}$ being

Received by the editors July 21, 2015 and, in revised form, August 26, 2016. 2010 Mathematics Subject Classification. Primary 11G05; Secondary 11F80. 
Laurent polynomials in those parameters. Also, Speyer 15 gave a combinatorial interpretation of the Somos sequences in terms of the number of perfect matchings in a family of graphs.

Somos-4 and Somos-5 type sequences are also connected with the arithmetic of elliptic curves (a connection made quite explicit by A. N. W. Hone in [5], and [6]). If $a_{n}$ is the $n$th term in the Somos-4 sequence, $E: y^{2}+y=x^{3}-x$ and $P=(0,0) \in E(\mathbb{Q})$, then the denominator of the $x$-coordinate of $(2 n-3) P$ is equal to $a_{n}^{2}$. It follows from this that $p \mid a_{n}$ if and only if $(2 n-3) P$ reduces to the identity in $E\left(\mathbb{F}_{p}\right)$, and so a prime $p$ divides a term in the Somos-4 sequence if and only if $(0,0) \in E\left(\mathbb{F}_{p}\right)$ has odd order. In $[8$, Rafe Jones and the third author prove that the density of primes dividing some term of the Somos- 4 sequence is $\frac{11}{21}$. The goal of the present paper is to prove an analogous result for the Somos-5 sequence.

Let $\pi^{\prime}(x)$ denote the number of primes $p \leq x$ so that $p$ divides some term in the Somos-5 sequence. We have the following table of data:

\begin{tabular}{c|cc}
$x$ & $\pi^{\prime}(x)$ & $\frac{\pi^{\prime}(x)}{\pi(x)}$ \\
\hline 10 & 3 & 0.750000 \\
$10^{2}$ & 12 & 0.480000 \\
$10^{3}$ & 83 & 0.494048 \\
$10^{4}$ & 588 & 0.478438 \\
$10^{5}$ & 4539 & 0.473207 \\
$10^{6}$ & 37075 & 0.472305 \\
$10^{7}$ & 314485 & 0.473209 \\
$10^{8}$ & 2725670 & 0.473087 \\
$10^{9}$ & 24057711 & 0.473134 \\
$10^{10}$ & 215298607 & 0.473129 \\
$10^{11}$ & 1948329818 & 0.473119
\end{tabular}

Our main result is the following.

Theorem 1. We have

$$
\lim _{x \rightarrow \infty} \frac{\pi^{\prime}(x)}{\pi(x)}=\frac{5087}{10752} \approx 0.473121 .
$$

The Somos- 5 sequence is related to the coordinates of rational points on the elliptic curve $E: y^{2}+x y=x^{3}+x^{2}-2 x$. This curve has $E(\mathbb{Q}) \cong \mathbb{Z} \times \mathbb{Z} / 2 \mathbb{Z}$ and generators are $P=(2,2)$ (of infinite order) and $Q=(0,0)$ (of order 2 ). We have (see Lemma 3) that

$$
m P+Q=\left(\frac{a_{m+2}^{2}-a_{m} a_{m+4}}{a_{m+2}^{2}}, \frac{4 a_{m} a_{m+2} a_{m+4}-a_{m}^{2} a_{m+6}-a_{m+2}^{3}}{a_{m+2}^{3}}\right) .
$$

It follows that a prime $p$ divides a term in the Somos- 5 sequence if and only if the reduction of $Q$ modulo $p$ is in $\langle P\rangle \subseteq E\left(\mathbb{F}_{p}\right)$. Another way of stating this is the following: there is a 2-isogeny $\phi: E \rightarrow E^{\prime}$, where $E^{\prime}: y^{2}+x y=x^{3}+x^{2}+8 x+10$ and

$$
\phi(x, y)=\left(\frac{x^{2}-2}{x}, \frac{x^{2} y+2 x+2 y}{x^{2}}\right) .
$$

The kernel of $\phi$ is $\{0, Q\}$. Letting $R=\phi(P)$ we show (see Theorem 4) that a prime $p$ of good reduction divides some term in the Somos-5 sequence if and only if the order of $P$ in $E\left(\mathbb{F}_{p}\right)$ is twice that of $R$ in $E^{\prime}\left(\mathbb{F}_{p}\right)$. 
A result of Pink (see Proposition 3.2 on page 284 of [11]) shows that the $\ell$-adic valuation of the order of a point $P(\bmod p)$ can be determined from a suitable Galois representation attached to an elliptic curve. For a positive integer $k$, we let $K_{k}$ be the field obtained by adjoining to $\mathbb{Q}$ the $x$ and $y$ coordinates of all points $\beta_{k}$ with $2^{k} \beta_{k}=P$. There is a Galois representation $\rho_{E, 2^{k}}: \operatorname{Gal}\left(K_{k} / \mathbb{Q}\right) \rightarrow$ $\mathrm{AGL}_{2}\left(\mathbb{Z} / 2^{k} \mathbb{Z}\right)$ and we relate the power of 2 dividing the order of $P$ in $E\left(\mathbb{F}_{p}\right)$ to $\rho_{E, 2^{k}}\left(\sigma_{p}\right)$, where $\sigma_{p}$ is a Frobenius automorphism at $p$ in $\operatorname{Gal}\left(K_{k} / \mathbb{Q}\right)$. Using the isogeny $\phi$ we are able to relate $\rho_{E, 2^{k}}\left(\sigma_{p}\right)$ and $\rho_{E^{\prime}, 2^{k-1}}\left(\sigma_{p}\right)$, obtaining a criterion that indicates when $p$ divides some term in the Somos- 5 sequence. We then determine the image of $\rho_{E, 2^{k}}$ for all $k$.

Once the image of $\rho_{E, 2^{k}}$ is known, the problem of computing the fraction of elements in the image with the desired properties is quite a difficult one. We introduce a new and simple method for computing this fraction and apply it to prove Theorem 1 .

\section{BACKGROUND}

If $E / F$ is an elliptic curve given in the form $y^{2}+a_{1} x y+a_{3} y=x^{3}+a_{2} x^{2}+a_{4} x+a_{6}$, the set $E(F)$ has the structure of an abelian group. Specifically, if $P, Q \in E(F)$, let $R=(x, y)$ be the third point of intersection between $E$ and the line through $P$ and $Q$. We define $P+Q=\left(x,-y-a_{1} x-a_{3}\right)$. The multiplication by $m$ map on an elliptic curve has degree $m^{2}$, and so if $E / \mathbb{C}$ is an elliptic curve and $\alpha \in E(\mathbb{C})$, then there are $m^{2}$ points $\beta$ so that $m \beta=\alpha$.

If $K / \mathbb{Q}$ is a finite extension, let $\mathcal{O}_{K}$ denote the ring of algebraic integers in $K$. A prime $p$ ramifies in $K$ if $p \mathcal{O}_{K}=\prod_{i=1}^{r} \mathfrak{p}_{i}^{e_{i}}$ and some $e_{i}>1$, where the $\mathfrak{p}_{i}$ are distinct prime ideals of $\mathcal{O}_{K}$.

Suppose $K / \mathbb{Q}$ is Galois, $p$ is a prime number that does not ramify in $K$, and $p \mathcal{O}_{K}=\prod_{i=1}^{g} \mathfrak{p}_{i}$. For each $i$, there is a unique element $\sigma \in \operatorname{Gal}(K / \mathbb{Q})$ for which

$$
\sigma(\alpha) \equiv \alpha^{p} \quad\left(\bmod \mathfrak{p}_{i}\right)
$$

for all $\alpha \in \mathcal{O}_{K}$. This element is called the Artin symbol of $\mathfrak{p}_{i}$ and is denoted $\left[\frac{K / \mathbb{Q}}{\mathfrak{p}_{i}}\right]$. If $i \neq j,\left[\frac{K / \mathbb{Q}}{\mathfrak{p}_{i}}\right]$ and $\left[\frac{K / \mathbb{Q}}{\mathfrak{p}_{j}}\right]$ are conjugate in $\operatorname{Gal}(K / \mathbb{Q})$ and $\left[\frac{K / \mathbb{Q}}{p}\right]:=$ $\left\{\left[\frac{K / \mathbb{Q}}{\mathfrak{p}_{i}}\right]: 1 \leq i \leq g\right\}$ is a conjugacy class in $\operatorname{Gal}(K / \mathbb{Q})$.

The key tool we will use in the proof of Theorem 1 is the Chebotarev density theorem.

Theorem 2 ([7], page 143$)$. If $C \subseteq \operatorname{Gal}(K / \mathbb{Q})$ is a conjugacy class, then

$$
\lim _{x \rightarrow \infty} \frac{\#\left\{p \leq x: p \text { prime },\left[\frac{K / \mathbb{Q}}{p}\right]=C\right\}}{\pi(x)}=\frac{|C|}{|\operatorname{Gal}(K / \mathbb{Q})|} .
$$

Roughly speaking, each element of $\operatorname{Gal}(K / \mathbb{Q})$ arises as $\left[\frac{K / \mathbb{Q}}{\mathfrak{p}}\right]$ equally often.

Let $E[m]=\{P \in E: m P=0\}$ be the set of points of order dividing $m$ on $E$. Then $\mathbb{Q}(E[m]) / \mathbb{Q}$ is Galois and $\operatorname{Gal}(\mathbb{Q}(E[m]) / \mathbb{Q})$ is isomorphic to a subgroup of $\operatorname{Aut}(E[m]) \cong \mathrm{GL}_{2}(\mathbb{Z} / m \mathbb{Z})$. Moreover, Proposition V.2.3 of [13] implies that if $\sigma_{p}$ is a Frobenius automorphism at some prime above $p$ and $\tau$ : $\operatorname{Gal}(\mathbb{Q}(E[m]) / \mathbb{Q}) \rightarrow \mathrm{GL}_{2}(\mathbb{Z} / m \mathbb{Z})$ is the usual $\bmod m$ Galois representation, then $\operatorname{tr} \tau\left(\sigma_{p}\right) \equiv p+1-\# E\left(\mathbb{F}_{p}\right)(\bmod m)$ and $\operatorname{det}\left(\tau\left(\sigma_{p}\right)\right) \equiv p(\bmod m)$. Another useful fact is the following. If $K / \mathbb{Q}$ is a number field, $\mathfrak{p}$ is a prime ideal in $\mathcal{O}_{K}$ above $p$, 
$\operatorname{gcd}(m, p)=1$ and $P \in E(K)[m]$ is not the identity, then $P$ does not reduce to the identity in $E\left(\mathcal{O}_{K} / \mathfrak{p}\right)$. This is a consequence of Proposition VII.3.1 of 13 .

We will construct Galois representations attached to elliptic curves with images in $\mathrm{AGL}_{2}\left(\mathbb{Z} / 2^{k} \mathbb{Z}\right) \cong\left(\mathbb{Z} / 2^{k} \mathbb{Z}\right)^{2} \rtimes \mathrm{GL}_{2}\left(\mathbb{Z} / 2^{k} \mathbb{Z}\right)$. Elements of such a group can be thought of either as pairs $(\vec{v}, M)$, where $\vec{v}$ is a row vector, and $M \in \mathrm{GL}_{2}\left(\mathbb{Z} / 2^{k} \mathbb{Z}\right)$, or as $3 \times 3$ matrices $\left[\begin{array}{lll}a & b & 0 \\ c & d & 0 \\ e & f & 1\end{array}\right]$, where $\vec{v}=\left[\begin{array}{ll}e & f\end{array}\right]$ and $M=\left[\begin{array}{ll}a & b \\ c & d\end{array}\right]$. In the former notation, the group operation is given by

$$
\left(\vec{v}_{1}, M_{1}\right) *\left(\vec{v}_{2}, M_{2}\right)=\left(\vec{v}_{1}+\vec{v}_{2} M_{1}, M_{2} M_{1}\right) .
$$

\section{Connection Between the Somos-5 Sequence And $E$}

Lemma 3. Define $P=(2,2)$ and $Q=(0,0)$ on $E: y^{2}+x y=x^{3}+x^{2}-2 x$. For all $m \geq 0$, we have the following relationship between the Somos-5 sequence and $E$ :

$$
m P+Q=\left(\frac{a_{m+2}^{2}-a_{m} a_{m+4}}{a_{m+2}^{2}}, \frac{4 a_{m} a_{m+2} a_{m+4}-a_{m}^{2} a_{m+6}-a_{m+2}^{3}}{a_{m+2}^{3}}\right) .
$$

Proof. We will prove this by strong induction. A straightforward calculation shows that the base cases $m=0$ and $m=1$ are true. For simplicity's sake, we will denote $a=a_{m}, b=a_{m+1}, c=a_{m+2}, d=a_{m+3}, e=a_{m+4}, f=a_{m+5}, g=a_{m+6}$, and $i=a_{m+8}$. Our inductive hypothesis is that

$$
m P+Q=\left(\frac{c^{2}-a e}{c^{2}}, \frac{4 a c e-a^{2} g-c^{3}}{c^{3}}\right) .
$$

We will now compute $(m+2) P+Q$.

To find the $x$ and $y$ coordinates of $(m+2) P+Q$, we add $2 P=(1,-1)$ to $m P+Q$. If $w$ is the slope and $v$ is the $y$-intercept, the line between $2 P$ and $m P+Q$ is $y=w x+v$ with $w=\frac{a g-4 c e}{c e}$ and $v=\frac{-a g+3 c e}{c e}$. Substituting this into the equation for $E$, we find the $x$-coordinate of $2 P+(m P+Q)$ to be $r_{x}=\frac{a^{2} g^{2}-7 a c e g+a e^{3}+9 c^{2} e^{2}}{c^{2} e^{2}}$. A straightforward but lengthy inductive calculation shows that if

$$
F(a, c, e, g)=a^{2} g^{2}-7 a c e g+a e^{3}+c^{3} g+8 c^{2} e^{2},
$$

then $F\left(a_{n}, a_{n+2}, a_{n+4}, a_{n+6}\right)=0$ for all $n$. Also, $a i=c g+8 e^{2}$ holds (by Proposition 2.8 in Hone's paper [6]). Since $F(a, c, e, g)=0$, we know that $r_{x}-\frac{F(a, c, e, g)}{c^{2} e^{2}}=r_{x}$. Therefore, we know that $r_{x}=\frac{-c g+e^{2}}{e^{2}}$.

Denote the $y$-coordinate of $(m+2) P+Q$ as $r_{y}$. We compute that $r_{y}=\frac{g(a g-3 c e)}{e^{3}}$. Using that $r_{y}=r_{y}-\frac{F(a, c, e, g)}{a e^{3}}$, we find that $r_{y}=\frac{4 c e g-c^{2} i-e^{3}}{e^{3}}$. Therefore, it is evident that

$$
(m+2) P+Q=\left(\frac{a_{m+4}^{2}-a_{m+2} a_{m+6}}{a_{m+4}^{2}}, \frac{4 a_{m+2} a_{m+4} a_{m+6}-a_{m+2}^{2} a_{m+8}-a_{m+4}^{3}}{a_{m+4}^{3}}\right) .
$$

Let $E^{\prime}$ be given by $E^{\prime}: y^{2}+x y=x^{3}+x^{2}+8 x+10$ and let $R=(1,4) \in E^{\prime}(\mathbb{Q})$. We have a 2-isogeny $\phi: E \rightarrow E^{\prime}$ given by

$$
\phi(x, y)=\left(\frac{x^{2}-2}{x}, \frac{x^{2} y+2 x+2 y}{x^{2}}\right) .
$$


The elliptic curves $E$ and $E^{\prime}$ each have conductor $102=2 \cdot 3 \cdot 17$. The next result classifies the primes of good reduction that divide a term in the Somos- 5 sequence.

Theorem 4. If $p$ is a prime of good reduction that divides a term in the Somos-5 sequence, the order of $P=(2,2)$ in $E\left(\mathbb{F}_{p}\right)$ is twice the order of $R=(1,4)$ in $E^{\prime}\left(\mathbb{F}_{p}\right)$. Otherwise, their orders are the same.

Proof. If $p$ divides a term in our sequence, say $a_{m}$, we know from our previous lemma that the denominators $(m-2) P+Q$ are divisible by $p$. Therefore, modulo $p$, $(m-2) P+Q=0$. The point $Q$ has order 2 , so adding $Q$ to both sides we know that $(m-2) P=Q$. Therefore, we can deduce that $Q \in\langle P\rangle$. We have $\operatorname{ker}(\phi)=\{Q, 0\}$ (see Section 3.4 of [14]). Therefore, if $\phi$ is restricted to the subgroup generated by $P$, we have $|\operatorname{ker}(\phi)|=2$. Since $\phi(P)=R$, by the first isomorphism theorem for groups, $\frac{|\langle P\rangle|}{|\operatorname{ker}(\phi)|}=|\langle R\rangle|$. It follows that $|P|=2 \cdot|R|$.

Alternatively, assume $p$ does not divide a term in the Somos-5 sequence. So, there is no $m$ such that $m P+Q=0$ modulo $p$, which implies that $Q \notin\langle P\rangle$. Therefore, the kernel of $\phi$ restricted to $\langle P\rangle$ is $\{0\}$ and so $|P|=|\phi(P)|=|R|$.

It is easy to see that 2 and 3 each divide terms in the Somos- 5 sequence, and the proof above can be modified to handle the case of 17 . In particular, 17 divides a term in the Somos-5 sequence if and only if $Q \in\langle P\rangle \subseteq E_{\mathrm{ns}}\left(\mathbb{F}_{17}\right)$. Since $E$ has non-split multiplicative reduction at 17 , we have an isomorphism $E_{\mathrm{ns}}\left(\mathbb{F}_{289}\right) \cong \mathbb{F}_{289}^{\times}$ (by Proposition III.2.5 of [13]). The image of $P$ in $\mathbb{F}_{289}^{\times}$has order 9 . Thus, $\langle P\rangle \subseteq$ $E_{\mathrm{ns}}\left(\mathbb{F}_{17}\right)$ has odd order and so $(0,0)$ cannot be contained in it. Thus, 17 does not divide any term in the Somos-5 sequence.

\section{Galois Representations}

Denote by $E\left[2^{r}\right]$ the set of points on $E$ with order dividing $2^{r}$. Denote $K_{r}$ as the field obtained by adjoining to $\mathbb{Q}$ all $x$ and $y$ coordinates of points $\beta$ with $2^{r} \beta=P$. For a prime $p$ that is unramified in $K_{r}$, let $\sigma=\left[\frac{K_{r} / \mathbb{Q}}{\mathfrak{p}_{i}}\right]$ for some prime ideal $\mathfrak{p}_{i}$ above $p$. Given a basis $\langle A, B\rangle$ for $E\left[2^{r}\right]$, for any such $\sigma \in \operatorname{Gal}\left(K_{r} / \mathbb{Q}\right)$, we have $\sigma(\beta)=\beta+e A+f B$. Also, $\sigma(A)=a A+b B$ and $\sigma(B)=c A+d B$. Define the map $\rho_{E, 2^{k}}: \operatorname{Gal}\left(K_{r} / \mathbb{Q}\right) \rightarrow \mathrm{AGL}_{2}\left(\mathbb{Z} / 2^{k} \mathbb{Z}\right)$ by $\rho_{E, 2^{k}}(\sigma)=(\vec{v}, M)$ where $M=\left[\begin{array}{ll}a & b \\ c & d\end{array}\right]$ and $\vec{v}=\left[\begin{array}{ll}e & f\end{array}\right]$. Let $\tau: \operatorname{Gal}\left(K_{r} / \mathbb{Q}\right) \rightarrow \mathrm{GL}_{2}\left(\mathbb{Z} / 2^{k} \mathbb{Z}\right)$ be given by $\tau(\sigma)=M$. In a similar way, we let $K_{r}^{\prime}$ be the field obtained by adjoining to $\mathbb{Q}$ the $x$ and $y$ coordinates of points $\beta^{\prime}$ with $2^{k} \beta^{\prime}=R$ and from this construct $\rho_{E^{\prime}, 2^{k}}: \operatorname{Gal}\left(K_{r}^{\prime} / \mathbb{Q}\right) \rightarrow \mathrm{AGL}_{2}\left(\mathbb{Z} / 2^{k} \mathbb{Z}\right)$.

Let $S=\{\beta \in E(\mathbb{C}): m \cdot \beta \in E(K)\}$ and let $L$ be the field obtained by adjoining all $x$ and $y$ coordinates of points in $S$ to $K$. Then the only primes $p$ that ramify in $L / K$ are those that divide $m$ and those where $E / K$ has bad reduction (see Proposition VIII.1.5(b) in [13]).

Note that, if $p$ is unramified, there are multiple primes $\mathfrak{p}_{i}$ above $p$ which could result in different matrices $M_{i}$ and $\vec{v}_{i}$. However, properties we consider of these $\vec{v}_{i}$ and $M_{i}$ do not depend on the specific choice of $\mathfrak{p}_{i}$. The map depends on the choice of basis for $E\left[2^{r}\right]$, we choose this basis as described below in Theorem 7 .

Let $\beta_{r} \in E(\mathbb{C})$ be a point with $2^{r} \beta_{r}=P$. We say that $\beta_{r}$ is an $r$ th preimage of $P$ under multiplication by 2 . Let $p$ be a prime with $p \neq 2,3$ or $17, \sigma=\left[\frac{K_{r} / \mathbb{Q}}{\mathfrak{p}_{i}}\right]$, 
and $(\vec{v}, M)=\rho_{E, 2^{r}}(\sigma)$. Assume that $\operatorname{det}(I-M) \not \equiv 0\left(\bmod 2^{r}\right)$. This implies that $\# E\left(\mathbb{F}_{p}\right) \not \equiv 0\left(\bmod 2^{r}\right)$.

Theorem 5. Assume the notation above. Then $2^{h} P$ has odd order in $E\left(\mathbb{F}_{p}\right)$ if and only if $2^{h} \vec{v}$ is in the image of $I-M$.

Proof. First, assume $2^{h} \vec{v}$ is in the image of $I-M$. This means that $\vec{x}=2^{h} \vec{v}+\vec{x} M$ for some row vector $\vec{x}$ with coordinates in $\left(\mathbb{Z} / 2^{r} \mathbb{Z}\right)^{2}$. If this is true for $\vec{x}=\left[\begin{array}{ll}e & f\end{array}\right]$, define $C:=2^{h} \beta_{r}+e A+f B \in E\left(K_{r}\right)$. Then $\sigma(C)=C$. Since $\mathcal{O}_{K_{r}} / \mathfrak{p}_{i}$ is an extension of $\mathbb{F}_{p}$, we can consider the reductions, modulo $\mathfrak{p}_{i}$, of $\beta_{r}, A, B$ and $P$, namely $\bar{\beta}_{r}$, $\bar{A}, \bar{B}$, and $\bar{P}$. Since $\sigma(C)=C$, we have that $\bar{C}=2^{h} \bar{\beta}_{r}+e \bar{A}+f \bar{B} \in E\left(\mathbb{F}_{p}\right)$ has the property that $2^{r} \bar{C}=2^{h} \bar{P}$.

If $|\bar{C}|$ is odd, then $\left|2^{h} \bar{P}\right|$ is necessarily odd. On the other hand, if $|\bar{C}|$ is even, then every multiplication of $C$ by 2 cuts the order by a factor of 2 until we arrive at a point of odd order. Since $\left|E\left(\mathbb{F}_{p}\right)\right| \equiv \operatorname{det}(I-M) \not \equiv 0\left(\bmod 2^{r}\right)$, the power of 2 dividing $|C|$ is also less than $r$, and so $\left|2^{r} \bar{C}\right|=\left|2^{h} \bar{P}\right|$ is odd.

Conversely, assume that $\left|2^{h} \bar{P}\right|$ is odd. Let $a$ be the multiplicative inverse of $2^{r}$ modulo $\left|2^{h} \bar{P}\right|$ and define $\bar{C}:=a 2^{h} \bar{P} \in E\left(\mathbb{F}_{p}\right)$. Then $2^{r} \bar{C}=2^{h} \bar{P}$ and so we have $2^{r}\left(\bar{C}-2^{h} \bar{\beta}_{r}\right)=0$, where $\beta_{r} \in E\left(K_{r}\right)$ and $\bar{\beta}_{r}$ is its reduction in $E(\mathbb{F})$, where $\mathbb{F} / \mathbb{F}_{p}$ is a finite extension.

It follows that $\bar{C}:=2^{h} \bar{\beta}_{r}+y \bar{A}+z \bar{B} \in E\left(\mathbb{F}_{p}\right)$ for some $y, z \in \mathbb{Z} / 2^{r} \mathbb{Z}$. Hence if we set $C:=2^{h} \beta_{r}+y A+z B$, then there is a Frobenius automorphism $\sigma \in \operatorname{Gal}\left(K_{r} / \mathbb{Q}\right)$ for which $\sigma(C) \equiv C\left(\bmod \mathfrak{p}_{i}\right)$ for any prime ideal $\mathfrak{p}_{i}$ above $p$.

We claim that $\sigma(C)=C$ (as elements of $E\left(K_{r}\right)$ ). Note that $\sigma(C)-C \in E\left[2^{r}\right]$ and $\sigma(C)-C$ reduces to the identity modulo $\mathfrak{p}_{i}$. Since reduction is injective on torsion points of order coprime to the characteristic, and $p$ is odd, it follows that $\sigma(C)=C$. It follows that if $\rho_{E, 2^{r}}(\sigma)=(\vec{v}, M)$, then $2^{h} \vec{v}=\left[\begin{array}{ll}y & z\end{array}\right](I-M)$, which implies that $2^{h} \vec{v}$ is in the image of $I-M$.

The following corollary is immediate.

Corollary 6. Let o be the smallest positive integer so that $2^{\circ} \vec{v}=\vec{x}(I-M)$ for some $\vec{x}$ with entries in $\left(\mathbb{Z} / 2^{r} \mathbb{Z}\right)^{2}$. Then $2^{o}$ is the highest power of 2 dividing $|P|$.

The following theorem gives a convenient choice of basis for $E\left[2^{k}\right]$ and $E^{\prime}\left[2^{k}\right]$.

Theorem 7. Given a positive integer $k$, there are points $A_{k}, B_{k} \in E(\mathbb{C})$ that generate $E\left[2^{k}\right]$ and points $C_{k}, D_{k} \in E^{\prime}(\mathbb{C})$ that generate $E^{\prime}\left[2^{k}\right]$ so that $\phi\left(A_{k}\right)=C_{k}$ and $\phi\left(B_{k}\right)=2 D_{k}$. These points also satisfy the relations:

$$
2 A_{k}=A_{k-1}, \quad 2 B_{k}=B_{k-1}, \quad 2 C_{k}=C_{k-1}, \text { and } 2 D_{k}=D_{k-1} .
$$

Proof. We will prove this by induction. Recall that $\phi: E \rightarrow E^{\prime}$ is the isogeny with ker $\phi=\{0, T\}$ where $T=(0,0)$. Let $\phi^{\prime}: E^{\prime} \rightarrow E$ be the dual isogeny, and note that $\phi \circ \phi^{\prime}(P)=2 P$. Base Case: Let $k=1$. We want to find $\left\langle A_{1}, B_{1}\right\rangle$ to generate $E[2]$ and $\left\langle C_{1}, D_{1}\right\rangle$ to generate $E^{\prime}[2]$ so that $\phi\left(A_{1}\right)=C_{1}$ and $\phi\left(B_{1}\right)=2 D_{1}$. We set $B_{1}=(0,0)$, and choose $A_{1}$ to be any non-identity point in $E[2]$ other than $(0,0)$. We set $C_{1}=\phi\left(A_{1}\right)=(-5 / 4,5 / 8)$ and choose $D_{1}$ to be any non-identity point in $E^{\prime}[2]$ other than $C_{1}$. Note that $\phi^{\prime}\left(D_{1}\right)=B_{1}$.

Inductive Hypothesis: Assume $\left\langle A_{k}, B_{k}\right\rangle=E\left[2^{k}\right]$ and $\left\langle C_{k}, D_{k}\right\rangle=E^{\prime}\left[2^{k}\right]$ so that $\phi\left(A_{k}\right)=C_{k}, \phi\left(B_{k}\right)=2 D_{k}$, and $\phi^{\prime}\left(D_{k}\right)=B_{k}$. Moreover, $D_{k} \notin \phi\left(E\left[2^{k}\right]\right)$.

Since $|\operatorname{ker} \phi|=2$, we have that $\phi\left(E\left[2^{k+1}\right]\right) \supseteq E^{\prime}\left[2^{k}\right]$. Hence, we can choose $B_{k+1}$ so that $\phi\left(B_{k+1}\right)=D_{k}$. Then $2 B_{k+1}=\phi^{\prime}\left(\phi\left(B_{k+1}\right)\right)=\phi^{\prime}\left(D_{k}\right)=B_{k}$. We choose 
$D_{k+1}$ so that $\phi^{\prime}\left(D_{k+1}\right)=B_{k+1}$. Note that $2 D_{k+1}=\phi\left(B_{k+1}\right)=D_{k}$ and so $D_{k+1} \in$ $E^{\prime}\left[2^{k+1}\right]$. Now we pick $A_{k+1}$ so that $2 A_{k+1}=A_{k}$ and define $C_{k+1}=\phi\left(A_{k+1}\right)$.

By our Inductive Hypothesis, $\left\langle A_{k}, B_{k}\right\rangle=E\left[2^{k}\right]$. This implies that $\left\langle A_{k}\right\rangle \cap\left\langle B_{k}\right\rangle=$ 0 , which in turn implies that $\left\langle 2 A_{k+1}\right\rangle \cap\left\langle 2 B_{k+1}\right\rangle=0$. Let $C \in\left\langle A_{k+1}\right\rangle \cap\left\langle B_{k+1}\right\rangle$. Then, $C=a A_{k+1}=b B_{k+1}$. Because $|m g|=\frac{|g|}{\operatorname{gcd}(m,|g|)},|C|=\frac{2^{k+1}}{2^{\text {ord }_{2}(a)}}=\frac{2^{k+1}}{2^{\text {ord }_{2}(b)}}$, where $\operatorname{ord}_{2}(n)$ is the highest power of 2 dividing $n$, it follows that either $a$ and $b$ are both even, or they are both odd. If $a$ and $b$ are odd, then $|C|=2^{k+1}$ but $2 C \in\left\langle A_{k}\right\rangle \cap\left\langle B_{k}\right\rangle=0$, which is a contradiction. If $a$ and $b$ are even, then $C \in\left\langle A_{k}\right\rangle \cap\left\langle B_{k}\right\rangle=0$. It follows that $\left\langle A_{k+1}\right\rangle \cap\left\langle B_{k+1}\right\rangle=0$, which gives that $E\left[2^{k+1}\right]=\left\langle A_{k+1}, B_{k+1}\right\rangle$.

Now we show that $\left\langle C_{k+1}, D_{k+1}\right\rangle=E^{\prime}\left[2^{k+1}\right]$, by way of showing that $\left\langle C_{k+1}\right\rangle \cap$ $\left\langle D_{k+1}\right\rangle=0$. We have shown that $\left\langle A_{k+1}, B_{k+1}\right\rangle=E\left[2^{k+1}\right]$, and so $\phi\left(E\left[2^{k+1}\right]\right)=$ $\left\langle C_{k+1}, 2 D_{k+1}\right\rangle$. We want to show that $D_{k+1} \notin \phi\left(E\left[2^{k+1}\right]\right)$.

If $D_{k+1} \in \phi\left(E\left[2^{k+1}\right]\right)$, then $D_{k+1}=a C_{k+1}+2 b D_{k+1}$. So, $a C_{k+1}+(2 b-1) D_{k+1}=$ 0 . Since $(2 b-1)$ is odd, $(2 b-1) D_{k+1}$ has order dividing $2^{k+1}$. Hence, $a C_{k+1}$ has order dividing $2^{k+1}$. We can then see that

$$
\begin{aligned}
2 a C_{k+1}+2(2 b-1) D_{k+1} & =0 \\
a C_{k}+(2 b-1) D_{k} & =0 \\
\Longrightarrow a \equiv(2 b-1) & \equiv 0 \quad\left(\bmod 2^{k}\right),
\end{aligned}
$$

which is a contradiction. This implies that $\phi\left(E\left[2^{k+1}\right]\right)$ is an index 2 subgroup of $\left\langle C_{k+1}, D_{k+1}\right\rangle$ of order $2^{2 k+1}$, and so $\left\langle C_{k+1}, D_{k+1}\right\rangle=E^{\prime}\left[2^{k+1}\right]$. This proves the desired claim.

Recall the maps $\rho_{E, 2^{k}}: \operatorname{Gal}\left(K_{k} / \mathbb{Q}\right) \rightarrow \operatorname{AGL}_{2}\left(\mathbb{Z} / 2^{k} \mathbb{Z}\right)$ and $\tau: \operatorname{Gal}\left(K_{k} / \mathbb{Q}\right) \rightarrow$ $\mathrm{GL}_{2}\left(\mathbb{Z} / 2^{k} \mathbb{Z}\right)$, defined at the beginning of this section. In [12, an algorithm is given to compute the image of the 2-adic Galois representation $\tau$. Running this algorithm shows that the image of $\tau$ (up to conjugacy) is the index 6 subgroup of $\mathrm{GL}_{2}\left(\mathbb{Z} / 2^{k} \mathbb{Z}\right.$ ) generated by $\left[\begin{array}{ll}1 & 1 \\ 0 & 1\end{array}\right],\left[\begin{array}{ll}7 & 0 \\ 2 & 1\end{array}\right]$, and $\left[\begin{array}{ll}5 & 0 \\ 2 & 1\end{array}\right]$. Moreover, the subgroup generated by the aforementioned matrices is the unique conjugate that corresponds to the basis chosen in Theorem 7

Theorem 8. If $\rho_{E, 2^{k}}(\sigma)=(\vec{v}, M)$ where $\vec{v}=(e, f)$, then $e \equiv 0(\bmod 2)$ if and only if $\operatorname{det}(M) \equiv 1,7(\bmod 8)$.

Proof. We will show that $e \equiv 0(\bmod 2)$ and $\operatorname{det}(M) \equiv 1,7(\bmod 8)$ if and only if $\sigma(\sqrt{2})=\sqrt{2}$.

Let $\beta_{1}$ be a point in $E\left(K_{1}\right)$ so that $2 \beta_{1}=(2,2)$. We pick a basis $\left\langle A_{1}, B_{1}\right\rangle$ according to Theorem 7 . We have $\sigma\left(\beta_{1}\right)=\beta_{1}+e A_{1}+f B_{1}$, where $e, f \in \mathbb{Z} / 2 \mathbb{Z}$.

Let $\phi: E \rightarrow E^{\prime}$ be the usual isogeny and note that $B_{1} \in \operatorname{ker} \phi$. Thus, $\phi\left(\sigma\left(\beta_{1}\right)\right)=$ $\phi\left(\beta_{1}+e A_{1}+f B_{1}\right)=\phi\left(\beta_{1}\right)+e \phi\left(A_{1}\right)$. It follows that $e \equiv 0(\bmod 2)$ if and only if $\sigma\left(\phi\left(\beta_{1}\right)\right)=\phi\left(\sigma\left(\beta_{1}\right)\right)=\phi\left(\beta_{1}\right)$. A straightforward computation shows that the coordinates of $\phi\left(\beta_{1}\right)$ generate $\mathbb{Q}(\sqrt{2})$. It follows that $e \equiv 0(\bmod 2)$ if and only if $\sigma(\sqrt{2})=\sqrt{2}$.

Finally, suppose that $\sigma$ is the Artin symbol associated to a prime ideal $\mathfrak{p}$ above a rational prime $p$. By properties of the Weil pairing (see [13], Section III.8), we have that $\zeta_{2^{k}}=e^{2 \pi i / 2^{k}} \in \mathbb{Q}\left(E\left[2^{k}\right]\right)$, and that $\sigma\left(\zeta_{2^{k}}\right)=\zeta_{2^{k}}^{\operatorname{det}(M)}=\zeta_{2^{k}}^{p}$. Since 
$\sqrt{2}=\zeta_{8}+\zeta_{8}^{-1}$, it follows easily that $\sigma(\sqrt{2})=\sqrt{2} \Longleftrightarrow p \equiv 1,7(\bmod 8)$ and hence $\sigma(\sqrt{2})=\sqrt{2}$ if and only if $\operatorname{det}(M) \equiv 1,7(\bmod 8)$.

For $k \geq 3$, define $I_{k}$ to be the subgroup of $\mathrm{AGL}_{2}\left(\mathbb{Z} / 2^{k} \mathbb{Z}\right)$ whose elements are ordered pairs $\{(\vec{v}, M)\}$ where $\vec{v}=\left[\begin{array}{ll}e & f\end{array}\right]$, the reduction of $M \bmod 8$ is in the group generated by $\left[\begin{array}{ll}1 & 1 \\ 0 & 1\end{array}\right],\left[\begin{array}{ll}7 & 0 \\ 2 & 1\end{array}\right]$, and $\left[\begin{array}{ll}5 & 0 \\ 2 & 1\end{array}\right]$, and $e \equiv 0(\bmod 2)$ if and only if $\operatorname{det}(M) \equiv 1$ or $7(\bmod 8)$. By Theorem 8 and the discussion preceeding it, we know that the image of $\rho_{E, 2^{k}}: \operatorname{Gal}\left(K_{k} / \mathbb{Q}\right) \rightarrow \mathrm{AGL}_{2}\left(\mathbb{Z} / 2^{k} \mathbb{Z}\right)$ is contained in $I_{k}$.

We now aim to show that the image of $\rho_{E, 2^{k}}: \operatorname{Gal}\left(K_{k} / \mathbb{Q}\right) \rightarrow \mathrm{AGL}_{2}\left(\mathbb{Z} / 2^{k} \mathbb{Z}\right)$ is $I_{k}$ for $k \geq 3$. By [13] (page 105), if we have an elliptic curve $E: y^{2}=x^{3}+A x+B$, the division polynomial $\psi_{m} \in \mathbb{Z}[A, B, x, y]$ is determined recursively by:

$$
\begin{aligned}
\psi_{1} & =1, \psi_{2}=2 y, \psi_{3}=3 x^{4}+6 A x^{2}+12 B x-A^{2}, \\
\psi_{4} & =4 y\left(x^{6}+5 A x^{4}+20 B x^{3}-5 A^{2} x^{2}-4 A B x-8 B^{2}-A^{3}\right), \\
\psi_{2 m+1} & =\psi_{m+2} \psi_{m}^{3}-\psi_{m-1} \psi_{m+1}^{3}, \quad 2 y \psi_{2 m}=\psi_{m}\left(\psi_{m+2} \psi_{m-1}^{2}-\psi_{m-2} \psi_{m+1}^{2}\right) .
\end{aligned}
$$

We then define $\phi_{m}$ and $\omega_{m}$ as follows:

$$
\begin{aligned}
\phi_{m} & =x \psi_{m}^{2}-\psi_{m+1} \psi_{m-1}, \\
4 y \omega_{m} & =\psi_{m+2} \psi_{m-1}^{2}-\psi_{m-2} \psi_{m+1}^{2} .
\end{aligned}
$$

If $\Delta=-16\left(4 A^{3}+27 B^{2}\right) \neq 0$, then $\phi_{m}(x)$ and $\psi_{m}(x)^{2}$ are relatively prime. This also implies that, for $P=\left(x_{0}, y_{0}\right) \in E$,

$$
[m] P=\left(\frac{\phi_{m}(P)}{\psi_{m}(P)^{2}}, \frac{\omega_{m}(P)}{\psi_{m}(P)^{3}}\right) .
$$

Lemma 9. The map $\rho_{E, 8}: \operatorname{Gal}\left(K_{3}, \mathbb{Q}\right) \rightarrow \mathrm{AGL}_{2}(\mathbb{Z} / 8 \mathbb{Z})$ has image $I_{3}$.

Proof. The curve $E$ is isomorphic to $E_{2}: y^{2}=x^{3}-3267 x+45630$. The isomorphism that takes $E$ to $E_{2}$ takes $P=(2,2)$ on $E$ to $P_{2}=(87,648)$ on $E_{2}$.

We use division polynomials to construct a polynomial $f(x)$ whose roots are the $x$-coordinates of points $\beta_{3}$ on $E_{2}$ so that $8 \beta_{3}=P_{2}$. By the above formulas, $8 P_{2}=\left(\frac{\phi_{8}\left(P_{2}\right)}{\psi_{8}\left(P_{2}\right)^{2}}, \frac{\omega_{8}\left(P_{2}\right)}{\psi_{8}\left(P_{2}\right)^{3}}\right)$. Since $P_{2}=(87,648)$,

$$
f(x)=\phi_{8}\left(P_{2}\right)-87 \psi_{8}\left(P_{2}\right)^{2}=0
$$

will yield the equation with roots that satisfy our requirement. This is a degree 64 polynomial. By using Magma to compute the Galois group of $f(x)$, we find the order to be 8192. A simple calculation shows that $I_{3}$ has order 8192 and since $f(x)$ splits in $K_{3} / \mathbb{Q}$, we have that $\operatorname{Gal}\left(K_{3} / \mathbb{Q}\right) \cong I_{3}$.

To show that the image of $\rho_{E, 2^{k}}$ is $I_{k}$, we will consider the Frattini subgroup of $I_{k}$. This is the intersection of all maximal subgroups of $I_{k}$. Since $I_{k}$ is a 2-group, every maximal subgroup is normal and has index 2. It follows from this that if $g \in I_{k}$, then $g^{2} \in \Phi\left(I_{k}\right)$.

Lemma 10. For $3 \leq k, \Phi\left(I_{k}\right)$ contains all pairs $(\vec{v}, M)$ such that $\vec{v} \equiv \overrightarrow{0}(\bmod 4)$ and $M \equiv I(\bmod 8)$.

Proof. We begin by observing that for $r=k,(0, I) \in \Phi\left(I_{k}\right)$. We prove the result by backwards induction on $r$. 
Inductive Hypothesis: $\Phi\left(I_{k}\right)$ contains all pairs $(0, M), M \equiv I\left(\bmod 2^{r}\right)$. Write $g=I+2^{r-2} N$ for some $N \in M_{2}(\mathbb{Z} / 4 \mathbb{Z})$, and let $h=I+2^{r-1} N$. If $r \geq 5$, then a straightforward calculation shows that $(0, g) \in I_{k}$. So, $(0, g)^{2}=\left(0, g^{2}\right) \in \Phi\left(I_{k}\right)$. Therefore, for $r>3$,

$$
g^{2}=I+2^{r-1} N+2^{2 r-4} N^{2} \equiv h \quad\left(\bmod 2^{2 r-4}\right) .
$$

By the induction hypothesis, $\left(0, g^{2} h^{-1}\right) \in \Phi\left(I_{k}\right)$, and so $(0, h) \in \Phi\left(I_{k}\right)$.

So, for $k \geq r \geq 4$, all pairs $(0, M), M \equiv I\left(\bmod 2^{r}\right) \in \Phi\left(I_{k}\right)$. We will now construct $I_{4}$, compute $\Phi\left(I_{4}\right)$, and show that $\Phi\left(I_{4}\right) \supseteq\{(\vec{v}, M): \vec{v} \equiv 0(\bmod 8), M \equiv$ $I(\bmod 8)\}$. A computation with Magma shows that

$$
I_{4}=\left\langle\left[\begin{array}{lll}
1 & 1 & 0 \\
0 & 1 & 0 \\
0 & 0 & 1
\end{array}\right],\left[\begin{array}{lll}
7 & 0 & 0 \\
2 & 1 & 0 \\
0 & 0 & 1
\end{array}\right],\left[\begin{array}{lll}
5 & 0 & 0 \\
2 & 1 & 0 \\
1 & 0 & 1
\end{array}\right]\right\rangle .
$$

We then construct $\Phi\left(I_{4}\right)$ and then $\phi: \Phi\left(I_{4}\right) \rightarrow \mathrm{GL}_{3}(\mathbb{Z} / 8 \mathbb{Z})$ obtained by reducing the entries modulo 8 . We check that $\operatorname{ker} \phi$ has order 64 and this proves the desired claim about $\Phi\left(I_{4}\right)$.

Now, observe that if $\vec{v}_{1}=(2 x, 2 y)$, then $\left(\vec{v}_{1}, I\right) \in I_{k}$ and so $\left(2 \vec{v}_{1}, I\right)=\left(\vec{v}_{1}, I\right)^{2} \in$ $\Phi\left(I_{k}\right)$, and so $\Phi\left(I_{k}\right)$ contains all pairs $(\vec{v}, I)$ with $\vec{v} \equiv \overrightarrow{0}(\bmod 4)$. Finally, for any matrix $M \equiv I(\bmod 8)$, we have

$$
\left(\vec{v}_{1}, I\right) *(0, M)=\left(\vec{v}_{1}, M\right) \in \Phi\left(I_{k}\right)
$$

and this proves the desired claim.

Finally, we determine the image.

Theorem 11. The map $\rho_{E, 2^{k}}: \operatorname{Gal}\left(K_{k} / \mathbb{Q}\right) \rightarrow \mathrm{AGL}_{2}\left(\mathbb{Z} / 2^{k} \mathbb{Z}\right)$ has image $I_{k}$ for all $k \geq 3$.

Proof. If not, the image of $\rho_{E, 2^{k}}$ is contained in a maximal subgroup $M$ of $I_{k}$. Lemma 10 implies that $M$ contains the kernel of the map from $I_{k} \rightarrow I_{3}$, and so the image of $\rho_{E, 8}$ must lie in a maximal subgroup of $I_{3}$. This contradicts Lemma 9, and shows the image is $I_{k}$.

Now, we indicate the relationship between $\rho_{E, 2^{k}}$ and $\rho_{E^{\prime}, 2^{k}}$. Let $\sigma \in \operatorname{Gal}\left(K_{k} / \mathbb{Q}\right)$. If $\beta_{k}$ is chosen so $2^{k} \beta_{k}=P$, then

$$
\begin{aligned}
\sigma\left(A_{k}\right) & =a A_{k}+b B_{k}, \\
\sigma\left(B_{k}\right) & =c A_{k}+d B_{k}, \\
\sigma\left(\beta_{k}\right) & =\beta_{k}+e A_{k}+f B_{k} .
\end{aligned}
$$

Applying $\phi$ to these equations, we have

$$
\begin{aligned}
& \phi\left(\sigma\left(A_{k}\right)\right)=a C_{k}+2 b D_{k}=\sigma\left(\phi\left(A_{k}\right)\right)=\sigma\left(C_{k}\right), \\
& \phi\left(\sigma\left(B_{k}\right)\right)=c C_{k}+2 d D_{k}=\sigma\left(\phi\left(B_{k}\right)\right)=\sigma\left(2 D_{k}\right), \\
& \phi\left(\sigma\left(\beta_{k}\right)\right)=\phi\left(\beta_{k}\right)+e C_{k}+2 f D_{k}=\sigma\left(\phi\left(\beta_{k}\right)\right)=\sigma\left(\beta_{k}^{\prime}\right),
\end{aligned}
$$

where $2^{k} \beta_{k}^{\prime}=R$ on $E^{\prime}$. Using the relations from Theorem 7 , we have that $2 D_{k}=$ $D_{k-1}$ and $2 C_{k}=C_{k-1}$. This gives

$$
\begin{aligned}
\sigma\left(C_{k-1}\right) & =a C_{k-1}+2 b D_{k-1}, \\
\sigma\left(D_{k-1}\right) & =\frac{c}{2} C_{k-1}+d D_{k-1} .
\end{aligned}
$$


Thus, $\rho_{E^{\prime}, 2^{k-1}}(\sigma)=\left(\vec{v}^{\prime}, M^{\prime}\right) \in \mathrm{AGL}_{2}\left(\mathbb{Z} / 2^{k-1} \mathbb{Z}\right)$, where $\vec{v}^{\prime}=\left[\begin{array}{ll}e & 2 f\end{array}\right]$ and $M^{\prime}=$ $\left[\begin{array}{cc}a & 2 b \\ \frac{c}{2} & d\end{array}\right]$.

Let $(v, M)$ be a vector-matrix pair in $I_{k}$. Suppose that $o$ is the smallest nonnegative integer so that $2^{o} \vec{v}$ is in the image of $(I-M)$. Thus there are integers $c_{1}$ and $c_{2}$ (not necessarily unique) so that $2^{\circ} \vec{v}=c_{1} \vec{x}_{1}+c_{2} \vec{x}_{2}$, where $\vec{x}_{1}$ and $\vec{x}_{2}$ are the first and second rows of $I-M$.

Lemma 12. Assume that $\operatorname{det}(M-I) \not \equiv 0\left(\bmod 2^{k}\right)$. If $c_{1} \vec{x}_{1}+c_{2} \vec{x}_{2}=d_{1} \vec{x}_{1}+d_{2} \vec{x}_{2}$, then $c_{1} \equiv d_{1}(\bmod 2)$ and $c_{2} \equiv d_{2}(\bmod 2)$.

Proof. The assumption on $\operatorname{det}(M-I)$ implies that $\operatorname{ker}(M-I)$ has order dividing $2^{k-1}$. However, if $c_{1} \vec{x}_{1}+c_{2} \vec{x}_{2}=d_{1} \vec{x}_{1}+d_{2} \vec{x}_{2}$, then $\left[\begin{array}{ll}c_{1}-d_{1} & c_{2}-d_{2}\end{array}\right]$ is an element of $\operatorname{ker}(M-I)$. If $c_{1} \not \equiv d_{1}(\bmod 2)$ or $c_{2} \not \equiv d_{2}(\bmod 2)$, then this element has order $2^{k}$, which is a contradiction.

The above lemma makes it so we can speak of $c_{1} \bmod 2$ and $c_{2} \bmod 2$ unambigously. We now have the following result.

Theorem 13. Assume the notation above. Let $o^{\prime}$ be the smallest positive integer so that $2^{o^{\prime}} v^{\prime}$ is in the image of $I-M^{\prime}$. If $\operatorname{det}(M-I) \not \equiv 0\left(\bmod 2^{k-1}\right)$, then $o \neq o^{\prime}$ if and only if $c_{1}$ is even.

Proof. Let $\vec{y}_{1}$ and $\vec{y}_{2}$ be the first two rows of $I-M^{\prime}$. A straightforward calculation shows that if $2^{o} \vec{v}=c_{1} \vec{x}_{1}+c_{2} \vec{x}_{2}$, then $2^{o} \vec{v}^{\prime}=c_{1} \vec{y}_{1}+2 c_{2} \vec{y}_{2}$. If $c_{1}$ is even, then it follows that $2^{o-1} \vec{v}^{\prime}=\left(c_{1} / 2\right) \vec{y}_{1}+c_{2} \vec{y}_{2}$ and so $o \neq o^{\prime}$.

Conversely, if $o \neq o^{\prime}$, then $o^{\prime} \leq o-1$ and so $2^{o-1} \vec{v}^{\prime}=d_{1} \vec{y}_{1}+d_{2} \vec{y}_{2}$. We have then that

$$
2^{o} \vec{v} \equiv 2 d_{1} \vec{x}_{1}+d_{2} \vec{x}_{2} \quad\left(\bmod 2^{k-1}\right) .
$$

So if $\vec{x}=\left[\begin{array}{ll}2 d_{1} & d_{2}\end{array}\right]$ we have $\vec{x}(I-M) \equiv 2^{o} \vec{v}\left(\bmod 2^{k-1}\right)$. If there is a vector $\vec{x}^{\prime}$ with $\vec{x} \not \equiv \vec{x}^{\prime}(\bmod 2)$ so that $\vec{x}^{\prime}(I-M) \equiv 2^{o} \vec{v}\left(\bmod 2^{k-1}\right)$, then $\vec{x}-\vec{x}^{\prime}$ is in the kernel of $I-M\left(\bmod 2^{k-1}\right)$. However, the order of $\vec{x}-\vec{x}^{\prime}$ is $2^{k-1}$ and this contradicts the condition on the determinant. This proves the desired result.

\section{Proof of Theorem 1}

Theorem 4 states that a prime $p$ divides a term in the Somos- 5 sequence if and only if the order of $P=(2,2) \in E\left(\mathbb{F}_{p}\right)$ is different from the order of $R=(1,4) \in$ $E^{\prime}\left(\mathbb{F}_{p}\right)$. Recall that $o$, the power of two dividing the order of $P$, is the smallest positive integer such that $2^{o} \vec{v} \in \operatorname{im}(I-M)$, and $o^{\prime}$ is the power of two dividing the order of $R$.

For the remainder of the argument, we will consider elements of $I_{k}$ as $3 \times 3$ matrices $\left[\begin{array}{lll}a & b & 0 \\ c & d & 0 \\ e & f & 1\end{array}\right]$ and consider $M$ as the $3 \times 3$ matrix $\left[\begin{array}{lll}a & b & 0 \\ c & d & 0 \\ 0 & 0 & 0\end{array}\right]$. We let $I-M=\left[\begin{array}{lll}\alpha & \beta & 0 \\ \gamma & \delta & 0 \\ e & f & 0\end{array}\right]$ and define $A=\gamma f-\delta e, B=\alpha f-\beta e$, and $C=\alpha \delta-\beta \gamma$. We define $M_{3}^{0}\left(\mathbb{Z} / 2^{k} \mathbb{Z}\right)$ to be the set of $3 \times 3$ matrices with entries in $\mathbb{Z} / 2^{k} \mathbb{Z}$ whose third column is zero. We will use $\operatorname{ord}_{2}(r)$ to denote the highest power of 2 dividing $r$ for $r \in \mathbb{Z} / 2^{k} \mathbb{Z}$. If $r=0 \in \mathbb{Z} / 2^{k} \mathbb{Z}$, we will interpret $\operatorname{ord}_{2}(r)$ to have an undefined value, but we will declare the inequality $\operatorname{ord}_{2}(r) \geq k$ to be true. 
Suppose that $\operatorname{det}(I-M) \not \equiv 0\left(\bmod 2^{k-1}\right)$. We have $2^{o} \vec{v} \in \operatorname{im}(I-M)$ if and only if $c_{1} \vec{x}_{1}+c_{2} \vec{x}_{2}=2^{o} \vec{v}$, where $M=\left[\begin{array}{ll}a & b \\ c & d\end{array}\right], \vec{x}_{1}=\left[\begin{array}{ll}1-a & -b\end{array}\right]$, and $\vec{x}_{2}=\left[\begin{array}{ll}-c & 1-d\end{array}\right]$. We know that $o \neq o^{\prime}$ if and only if $c_{1}$ is even. Solving the equation $c_{1} \vec{x}_{1}+c_{2} \vec{x}_{2}=2^{o} \vec{v}$ using Cramer's rule gives that $c_{1} C=-2^{\circ} A$ and $c_{2} C=2^{\circ} B$. Assuming that $c_{1}$ is even and $o>0$ implies that $c_{2}$ must be odd. (If $c_{1}$ and $c_{2}$ are both even, then $2^{o-1} \vec{v}=\left(c_{1} / 2\right) \vec{x}_{1}+\left(c_{2} / 2\right) \vec{x}_{2}$, which contradicts the definition of $o$.) The fact that $c_{2}$ is odd, together with $c_{2} C=2^{o} B$ implies that $\operatorname{ord}_{2}(B)<\operatorname{ord}_{2}(C)$. Moreover, since the power of 2 dividing $c_{1} C$ must be higher than that of $c_{2} C$ it follows that $\operatorname{ord}_{2}(B)<\operatorname{ord}_{2}(A)$. Conversely, if $\operatorname{ord}_{2}(B)<\operatorname{ord}_{2}(A)$ and $\operatorname{ord}_{2}(B)<\operatorname{ord}_{2}(C)$, then $o>0$ and $c_{1}$ is even. Therefore, our goal is the counting of elements of $I_{k}$ with $\operatorname{ord}_{2}(A)>\operatorname{ord}_{2}(B)$ and $\operatorname{ord}_{2}(C)>\operatorname{ord}_{2}(B)$. For an $M_{0} \in M_{3}^{0}\left(\mathbb{Z} / 2^{r} \mathbb{Z}\right)$, define

$$
\begin{aligned}
\eta\left(M_{0}, r, k\right) & =\#\left\{M \in M_{3}^{0}\left(\mathbb{Z} / 2^{k} \mathbb{Z}\right): M \equiv M_{0} \quad\left(\bmod 2^{r}\right),\right. \\
\left.\operatorname{ord}_{2}(A), \operatorname{ord}_{2}(C)>\operatorname{ord}_{2}(B)\right\}, & \\
\mu\left(M_{0}, r\right) & =\lim _{k \rightarrow \infty} \frac{\eta\left(M_{0}, r, k\right)}{\left|I_{3}\right| \cdot 64^{k-3}} .
\end{aligned}
$$

Roughly speaking, $\mu\left(M_{0}, r\right)$ is the fraction of matrices $M \equiv M_{0}\left(\bmod 2^{r}\right)$ in $I_{k}$ with the property that $\rho_{E, 2^{k}}\left(\sigma_{p}\right)=M$ implies that $p$ divides a term of the Somos- 5 sequence.

Theorem 14. We have

$$
\lim _{x \rightarrow \infty} \frac{\pi^{\prime}(x)}{\pi(x)}=\sum_{M \in I_{3}} \mu(I-M, 3) .
$$

Before we start the proof, we need some lemmas. The first is straightforward, and we omit its proof.

Lemma 15. If $a \in \mathbb{Z} / 2^{k} \mathbb{Z}$, then the number of pairs $(x, y) \in\left(\mathbb{Z} / 2^{k} \mathbb{Z}\right)^{2}$ with $x y \equiv a$ $\left(\bmod 2^{k}\right)$ is $\left(\operatorname{ord}_{2}(a)+1\right) 2^{k-1}$, where if $a \equiv 0\left(\bmod 2^{k}\right)$, we take $\operatorname{ord}_{2}(a)=k+1$.

Lemma 16. The number of matrices $M \in M_{2}\left(\mathbb{Z} / 2^{k} \mathbb{Z}\right)$ with $\operatorname{det}(M) \equiv 0\left(\bmod 2^{k}\right)$ is $3 \cdot 2^{3 k-1}-2^{2 k-1}$.

Proof. We count quadruples $(a, b, c, d)$ with $a d \equiv b c\left(\bmod 2^{k}\right)$. By Lemma 15, this number is equal to

$$
\sum_{\alpha \in \mathbb{Z} / 2^{k} \mathbb{Z}}\left(\left(\operatorname{ord}_{2}(\alpha)+1\right) 2^{k-1}\right)^{2},
$$

which can easily be shown to equal $3 \cdot 2^{3 k-1}-2^{2 k-1}$.

Proof of Theorem 14, For $k \geq 1$, let $G=\operatorname{Gal}\left(K_{k} / \mathbb{Q}\right)$ and $\sigma \in G$ have the property that $\sigma=\left[\frac{K_{k} / \mathbb{Q}}{\mathfrak{p}}\right]$ for some prime ideal $\mathfrak{p} \subseteq O_{K_{k}}$ with $\mathfrak{p} \cap \mathbb{Z}=(p)$. Assume that $p$ is unramified in $K_{k} / \mathbb{Q}$ and $E / \mathbb{F}_{p}$ has good reduction at $p$. Let $M$ be the $3 \times 3$ matrix corresponding to $\rho_{E, 2^{k}}(\sigma)$, and $A, B$ and $C$ be the corresponding minors of $I-M$. Then one of three alternatives occurs:

(a) $B \not \equiv 0\left(\bmod 2^{k}\right)$, and a higher power of 2 divides both $A$ and $C$.

In this situation (the good case), previous results ensure that the order of $P$ in $E\left(\mathbb{F}_{p}\right)$ is twice the order of $R$ in $E^{\prime}\left(\mathbb{F}_{p}\right)$, and hence $p$ divides some term in the Somos-5 sequence. 
(b) One of $A$ or $C$ is not congruent to $0 \bmod 2^{k}$ and the power of 2 dividing $B$ is equal to or higher than for $A$ or $C$.

In this situation (the bad case), previous results ensure that the order of $P$ in $E\left(\mathbb{F}_{p}\right)$ is equal to the order of $R$ in $E^{\prime}\left(\mathbb{F}_{p}\right)$ and $p$ does not divide any term in the Somos-5 sequence.

(c) $A \equiv B \equiv C \equiv 0\left(\bmod 2^{k}\right)$.

In this situation (the inconclusive case), we do not have enough information to determine if $p$ divides a term in the Somos-5 sequence or not.

Fix $\epsilon>0$ and choose a $k$ large enough so that both of the following conditions are satisfied:

(i) $\left|\sum_{M \in I_{3}} \frac{\eta(I-M, 3, k)}{\left|I_{3}\right| 64^{k-3}}-\sum_{M \in I_{3}} \mu(I-M, 3)\right|<\epsilon / 3$, and

(ii) the fraction of elements $M$ in $I_{k}$ with $C \equiv \operatorname{det}(I-M) \equiv 0\left(\bmod 2^{k-1}\right)$ is less than $\epsilon / 3$. (A matrix $M \in M_{2}\left(\mathbb{Z} / 2^{k} \mathbb{Z}\right)$ has determinant $\equiv 0\left(\bmod 2^{k-1}\right)$ if and only if its reduction modulo $2^{k-1}$ has determinant $\equiv 0\left(\bmod 2^{k-1}\right)$. Thus, by Lemma 16, there are $16 \cdot\left(3 \cdot 2^{3(k-1)-1}-2^{2(k-1)-1}\right)$ such matrices. Thus, the fraction of such $M$ is $3 \cdot 2^{-3 k+5}-2^{-4 k+6} \rightarrow 0$ as $k \rightarrow \infty$.)

Let $\mathcal{C} \subseteq I_{k}$ be the collection of "good" elements of $I_{k}$ and let $\mathcal{C}^{\prime}$ be the collection of "good or inconclusive" elements.

By the statements above, we have that

$$
\sum_{M \in I_{3}} \mu(I-M, 3)-2 \epsilon / 3<\frac{|\mathcal{C}|}{\left|I_{k}\right|}
$$

and

$$
\frac{\left|\mathcal{C}^{\prime}\right|}{\left|I_{k}\right|}<\sum_{M \in I_{3}} \mu(I-M, 3)+\epsilon / 3
$$

By the Chebotarev density theorem, we have

$$
\lim _{x \rightarrow \infty} \frac{\#\left\{p \text { prime }: p \leq x \text { is unramified in } K_{k} \text { and }\left[\frac{K_{k} / \mathbb{Q}}{p}\right] \subseteq \mathcal{C}\right\}}{\pi(x)}=\frac{|\mathcal{C}|}{\left|I_{k}\right|},
$$

and the same with $\mathcal{C}^{\prime}$.

Let $r$ be the number of primes that either ramify in $K_{k} / \mathbb{Q}$ or for which $E / \mathbb{Q}$ has bad reduction. Then there is a constant $N$ so that if $x>N$, then

$$
\begin{aligned}
& \sum_{M \in I_{3}} \mu(I-M, 3)-\epsilon+\frac{r}{\pi(x)} \\
&<\frac{\#\left\{p \text { prime }: p \leq x \text { is unramified in } K_{k} \text { and }\left[\frac{K_{k} / \mathbb{Q}}{p}\right] \subseteq \mathcal{C}\right\}}{\pi(x)},
\end{aligned}
$$

and

$$
\begin{gathered}
\frac{\#\left\{p \text { prime }: p \leq x \text { is unramified in } K_{k} \text { and }\left[\frac{K_{k} / \mathbb{Q}}{p}\right] \subseteq \mathcal{C}^{\prime}\right\}}{\pi(x)} \\
<\sum_{M \in I_{3}} \mu(I-M, 3)+\epsilon-\frac{r}{\pi(x)} .
\end{gathered}
$$

It follows from these inequalities that for $x>N$, then

$$
-\epsilon<\frac{\pi^{\prime}(x)}{\pi(x)}-\sum_{M \in I_{3}} \mu(I-M, 3)<\epsilon .
$$


This proves that

$$
\lim _{x \rightarrow \infty} \frac{\pi^{\prime}(x)}{\pi(x)}=\sum_{M \in I_{3}} \mu(I-M, 3)
$$

Our goal is now to compute $\sum_{M \in I_{3}} \mu(I-M, 3)$. To do this, we will develop rules to compute $\mu(M, r)$ for any matrix $M \in M_{3}\left(\mathbb{Z} / 2^{r} \mathbb{Z}\right)$ whose third column is zero. Observe that $\mu\left(M_{0}, r\right) \leq \frac{\#\left\{M \in M_{3}^{0}\left(\mathbb{Z} / 2^{r} \mathbb{Z}\right): M \equiv M_{0}\left(\bmod 2^{r}\right)\right\}}{\left|I_{3}\right| \cdot 64^{r-3}}=\frac{1}{2 \cdot 64^{r-1}}$.

Also, if all the entires in $M$ are even, then $\mu(M, r)=\frac{1}{64} \mu\left(\frac{M}{2}, r-1\right)$. This allows us to reduce to matrices where at least one entry is odd. If $M \in M_{3}^{0}(\mathbb{Z} / 2 \mathbb{Z})$ is the zero matrix, we have

$$
\begin{aligned}
\mu(M, 1) & =\frac{1}{64} \mu(M / 2,0) \\
& =\frac{1}{64} \sum_{N \in M_{3}^{0}(\mathbb{Z} / 2 \mathbb{Z})} \mu(N, 1)=\frac{1}{64} \mu(M, 1)+\frac{1}{64} \sum_{\substack{N \in M_{3}^{0}(\mathbb{Z} / 2 \mathbb{Z}) \\
N \neq M}} \mu(N, 1) .
\end{aligned}
$$

It follows that $\mu(M, 1)=\frac{1}{63} \sum_{\substack{N \in M_{3}^{0}(\mathbb{Z} / 2 \mathbb{Z}) \\ N \neq M}} \mu(N, 1)$.

In order to determine $\mu\left(M_{0}, r\right)$, it is necessary to consider a matrix

$$
M \in M_{3}\left(\mathbb{Z} / 2^{k} \mathbb{Z}\right)
$$

and examine the behavior of matrices $M^{\prime} \in M_{3}\left(\mathbb{Z} / 2^{k+1} \mathbb{Z}\right)$ with $M^{\prime} \equiv M\left(\bmod 2^{k}\right)$. We refer to these as 'lifts' of $M$. We define $A, B$ and $C$ to be functions defined on a matrix $M=\left[\begin{array}{lll}\alpha & \beta & 0 \\ \gamma & \delta & 0 \\ e & f & 0\end{array}\right]$, given by $A=\gamma f-\delta e, B=\alpha f-\beta e$ and $C=\alpha \delta-\beta \gamma$.

Theorem 17. Let $k \geq 1$ and $M=\left[\begin{array}{lll}\alpha & \beta & 0 \\ \gamma & \delta & 0 \\ e & f & 0\end{array}\right] \in M_{3}^{0}\left(\mathbb{Z} / 2^{k} \mathbb{Z}\right)$ and suppose $A \equiv$ $B \equiv C \equiv 0\left(\bmod 2^{k}\right)$.

(1) If $\gamma$ or $\delta$ is odd, then $\mu(M, k)=0$.

(2) If $\gamma$ and $\delta$ are both even, but one of $\alpha, \beta$, e or $f$ is odd, then $\mu(M, k)=$ $\frac{1}{6 \cdot 64^{k-1}}$.

Proof. Consider $M^{\prime}$ to be a lift of $M \bmod 2^{k+1}$ and write

$$
M^{\prime}=\left[\begin{array}{lll}
\alpha^{\prime} & \beta^{\prime} & 0 \\
\gamma^{\prime} & \delta^{\prime} & 0 \\
e^{\prime} & f^{\prime} & 0
\end{array}\right]
$$

Assume that $\gamma$ is odd and $A^{\prime}=\gamma^{\prime} f^{\prime}-\delta^{\prime} e^{\prime} \equiv 0\left(\bmod 2^{k+1}\right)$ and $C^{\prime}=\alpha^{\prime} \delta^{\prime}-\beta^{\prime} \gamma^{\prime} \equiv 0$ $\left(\bmod 2^{k+1}\right)$. From this, we get that $f^{\prime} \equiv \frac{e^{\prime} \delta^{\prime}}{\gamma^{\prime}}\left(\bmod 2^{k}\right)$ and $\beta^{\prime} \equiv \frac{\alpha^{\prime} \delta^{\prime}}{\gamma^{\prime}}\left(\bmod 2^{k}\right)$. We then find that $B \equiv \alpha^{\prime} f^{\prime}-\beta^{\prime} e^{\prime} \equiv \alpha^{\prime}\left(\frac{e^{\prime} \delta^{\prime}}{\gamma^{\prime}}\right)-\left(\frac{\alpha^{\prime} \delta^{\prime}}{\gamma^{\prime}}\right) e^{\prime} \equiv 0\left(\bmod 2^{k}\right)$. It follows that none of the lifts of $M$ have $\operatorname{ord}_{2}(B)<\min \left\{\operatorname{ord}_{2}(A), \operatorname{ord}_{2}(C)\right\}$ and so $\mu(M, k)=0$. A similar argument applies in the case that $\delta$ is odd. 
Suppose now that $\gamma$ and $\delta$ are both even. In this case, write

$$
M^{\prime}=\left[\begin{array}{lll}
\alpha+\alpha_{1} 2^{k} & \beta+\beta_{1} 2^{k} & 0 \\
\gamma+\gamma_{1} 2^{k} & \delta+\delta_{1} 2^{k} & 0 \\
e+e_{1} 2^{k} & f+f_{1} 2^{k} & 0
\end{array}\right]
$$

where $\alpha_{1}, \beta_{1}, \gamma_{1}, \delta_{1}, e_{1}, f_{1} \in \mathbb{F}_{2}$. If $A^{\prime}, B^{\prime}$ and $C^{\prime}$ are the values of $A, B$, and $C$ associated to $M^{\prime}$, then

$$
\begin{array}{rc}
A^{\prime} \equiv A+2^{k}\left(\gamma_{1} f-\delta_{1} e\right) & \left(\bmod 2^{k+1}\right) \\
B^{\prime} \equiv B+2^{k}\left(\alpha_{1} f+\alpha f_{1}-\beta_{1} e-\beta e_{1}\right) & \left(\bmod 2^{k+1}\right) \\
C^{\prime} \equiv C+2^{k}\left(\alpha \delta_{1}-\beta \gamma_{1}\right) & \left(\bmod 2^{k+1}\right)
\end{array}
$$

Suppose that $e$ or $f$ is odd. Then the map $\mathbb{F}_{2}^{6} \rightarrow \mathbb{F}_{2}^{2}$ given by $\left(\alpha_{1}, \beta_{1}, \gamma_{1}, \delta_{1}, e_{1}, f_{1}\right)$ $\mapsto\left(\gamma_{1} f-\delta_{1} e, \alpha_{1} f+\alpha f_{1}-\beta_{1} e-\beta e_{1}\right)$ is surjective. It follows that of the 64 lifts of $M$, one quarter have $\left(A^{\prime} \bmod 2^{k+1}, B^{\prime} \bmod 2^{k+1}\right)$ equal to each of $\left(2^{k}, 2^{k}\right)$, $\left(0,2^{k}\right),\left(2^{k}, 0\right)$ and $(0,0)$. Moreover, if $A^{\prime} \equiv 0\left(\bmod 2^{k+1}\right)$, then we must have $C^{\prime} \equiv 0\left(\bmod 2^{k+1}\right)$. This is because if $e^{\prime}$ is odd, then $\delta^{\prime} \equiv \frac{\gamma^{\prime} f^{\prime}}{e^{\prime}}\left(\bmod 2^{k+1}\right)$, and $\beta^{\prime} \equiv \frac{\alpha^{\prime} f^{\prime}-B^{\prime}}{e^{\prime}}\left(\bmod 2^{k+1}\right)$. Plugging these into $C^{\prime}=\alpha^{\prime} \delta^{\prime}-\beta^{\prime} \gamma^{\prime}$ gives $C^{\prime} \equiv$ $\frac{B^{\prime} \gamma^{\prime}}{e^{\prime}}\left(\bmod 2^{k+1}\right)$. Since $\gamma^{\prime}$ is even, it follows that $C^{\prime} \equiv 0\left(\bmod 2^{k+1}\right)$. A similar argument shows that $C^{\prime} \equiv 0\left(\bmod 2^{k+1}\right)$ if $f^{\prime}$ is odd. As a consequence, of the 64 lifts of $M, 32$ have $\mu\left(M^{\prime}, k+1\right)=0,16$ have $\operatorname{ord}_{2}\left(B^{\prime}\right)<\operatorname{ord}_{2}\left(A^{\prime}\right)$ and $\operatorname{ord}_{2}\left(B^{\prime}\right)<$ $\operatorname{ord}_{2}\left(C^{\prime}\right)$. For these, we have $\mu\left(M^{\prime}, k+1\right)=\frac{1}{2 \cdot 64^{k}}$. The remainder have $A^{\prime} \equiv B^{\prime} \equiv$ $C^{\prime} \equiv 0\left(\bmod 2^{k+1}\right)$. It follows that

$$
\mu(M, k)=\frac{1}{2 \cdot 64^{k-1}} \cdot \frac{1}{4}+\sum_{\substack{M^{\prime} \equiv M \\ A^{\prime} \equiv B^{\prime} \equiv C^{\prime} \equiv 0 \quad\left(\bmod 2^{k+1}\right)}} \mu\left(M^{\prime}, k+1\right) .
$$

Applying the above argument repeatedly gives

$$
\mu(M, k)=\frac{1}{2 \cdot 64^{k-1}} \cdot\left(\frac{1}{4}+\frac{1}{16}+\cdots+\frac{1}{4^{\ell}}\right)+\sum_{\substack{M^{\prime} \equiv M \\ A^{\prime} \equiv B^{\prime} \equiv C^{\prime} \equiv 0\left(\bmod 2^{k+\ell}\right) \\\left(\bmod 2^{k+\ell}\right)}} \mu\left(M^{\prime}, k+\ell\right) .
$$

Using the bound $0 \leq \mu\left(M^{\prime}, k+\ell\right) \leq \frac{1}{2 \cdot 64^{k+\ell-1}}$, noting that the sum contains $16^{\ell}$ terms, and taking the limit as $\ell \rightarrow \infty$ yields that $\mu(M, k)=\frac{1}{2 \cdot 64^{k-1}} \sum_{r=1}^{\infty} \frac{1}{4^{r}}=$ $\frac{1}{6 \cdot 64^{k-1}}$.

The case when $\alpha$ or $\beta$ is odd is very similar. In that case, one can show that the 64 lifts $M^{\prime}$ have $\left(B^{\prime} \bmod 2^{k+1}, C^{\prime} \bmod 2^{k+1}\right)$ divided equally between $\left(2^{k}, 2^{k}\right),\left(0,2^{k}\right),\left(2^{k}, 0\right)$ and $(0,0)$, and that $C^{\prime} \equiv 0\left(\bmod 2^{k+1}\right)$ implies that $A^{\prime} \equiv 0$ $\left(\bmod 2^{k+1}\right)$. Again, one quarter of the lifts $M^{\prime}$ have $B^{\prime} \equiv 2^{k}\left(\bmod 2^{k+1}\right)$ and $A^{\prime} \equiv C^{\prime} \equiv 0\left(\bmod 2^{k+1}\right)$, and $\mu(M, k)=\frac{1}{6 \cdot 64^{k-1}}$.

Let $M \in M_{3}^{0}(\mathbb{Z} / 8 \mathbb{Z})$ be the zero matrix. We have that $\mu(M, 3)=\frac{1}{64^{2}} \mu(M, 1)=$ $\frac{1}{63} \cdot \frac{1}{64^{2}} \sum_{N \in M_{3}^{0}(\mathbb{Z} / 2 \mathbb{Z})} \mu(N, 1)$. Of the 63 non-zero matrices in $M_{3}^{0}(\mathbb{Z} / 2 \mathbb{Z})$ we find that 6 have $B$ odd and $A$ and $C$ even, while 36 have $A$ or $C$ odd. Of the remaining 21 , there are 12 that have $\gamma$ or $\delta$ odd, and the remaining 9 have $\gamma$ and $\delta$ both even. It follows that

$$
\mu(M, 3)=\frac{1}{63} \cdot \frac{1}{64^{2}} \cdot \frac{1}{2} \cdot\left[6+36 \cdot 0+12 \cdot 0+9 \cdot \frac{1}{3}\right]=\frac{1}{8192} \cdot \frac{1}{7}=\frac{1}{57344} .
$$


(Note that in the denominator of $\mu(N, 1)$ we have $\left|I_{3}\right| 64^{-2}=8192 \cdot(1 / 4096)=2$.)

For each of the 8191 non-identity elements $M$ of $I_{3}$, we divide $I-M$ by the highest power of 2 dividing all of the elements, say $2^{r}$. In 3754 cases, we have $\operatorname{ord}_{2}(B)<\operatorname{ord}_{2}(A)$ and $\operatorname{ord}_{2}(B)<\operatorname{ord}_{2}(C)$. For each of these, $\mu(I-M, 3)=\frac{1}{8192}$.

In 4036 cases, we have $\operatorname{ord}_{2}(B) \geq \operatorname{ord}_{2}(A)$ or $\operatorname{ord}_{2}(B) \geq \operatorname{ord}_{2}(C)$ and not all of $A, B$ and $C$ are congruent to 0 modulo $2^{3-r}$. For each of these, $\mu(I-M, 3)=0$.

In 365 cases, we have $A \equiv B \equiv C \equiv 0\left(\bmod 2^{3-r}\right)$ and $\gamma$ and $\delta$ are both even. In each of these cases, $\mu(I-M, 3)=\frac{1}{3 \cdot 8192}$ by Theorem 17

In the remaining 36 cases, we have $A \equiv B \equiv 0\left(\bmod 2^{3-r}\right)$ and one of $\gamma$ or $\delta$ is odd. By Theorem [17, $\mu(I-M, 3)=0$.

It follows that

$$
\sum_{M \in I_{3}} \mu(I-M, 3)=3754 \cdot \frac{1}{8192}+365 \cdot \frac{1}{3 \cdot 8192}+\frac{1}{57344}=\frac{5087}{10752} .
$$

This concludes the proof of Theorem 1

\section{ACKNOWLEDGMENTS}

The first and second authors thank the Wake Forest Undergraduate Research and Creative Activities Center for financial support. The authors used Magma 1] version 2.20-6 for computations. The authors would like to thank the anonymous referee for an especially thorough report with a number of suggestions that have improved the paper.

\section{REFERENCES}

[1] Wieb Bosma, John Cannon, and Catherine Playoust, The Magma algebra system. I. The user language, J. Symbolic Comput. 24 (1997), no. 3-4, 235-265, DOI 10.1006/jsco.1996.0125. Computational algebra and number theory (London, 1993). MR.1484478

[2] Paul Cubre and Jeremy Rouse, Divisibility properties of the Fibonacci entry point, Proc. Amer. Math. Soc. 142 (2014), no. 11, 3771-3785, DOI 10.1090/S0002-9939-2014-12269-6. MR.3251719

[3] Sergey Fomin and Andrei Zelevinsky, The Laurent phenomenon, Adv. in Appl. Math. 28 (2002), no. 2, 119-144, DOI 10.1006/aama.2001.0770. MR.1888840

[4] Helmut Hasse, Über die Dichte der Primzahlen p, für die eine vorgegebene ganzrationale Zahl $a \neq 0$ von gerader bzw.ungerader Ordnung mod. $p$ ist (German), Math. Ann. 166 (1966), 19-23, DOI 10.1007/BF01361432. MR0205975

[5] A. N. W. Hone, Elliptic curves and quadratic recurrence sequences, Bull. London Math. Soc. 37 (2005), no. 2, 161-171, DOI 10.1112/S0024609304004163. MR.2119015

[6] A. N. W. Hone, Sigma function solution of the initial value problem for Somos 5 sequences, Trans. Amer. Math. Soc. 359 (2007), no. 10, 5019-5034, DOI 10.1090/S0002-9947-07-04215-8. MR2320658

[7] Henryk Iwaniec and Emmanuel Kowalski, Analytic number theory, American Mathematical Society Colloquium Publications, vol. 53, American Mathematical Society, Providence, RI, 2004. MR2061214

[8] Rafe Jones and Jeremy Rouse, Galois theory of iterated endomorphisms, Proc. Lond. Math. Soc. (3) 100 (2010), no. 3, 763-794, DOI 10.1112/plms/pdp051. Appendix A by Jeffrey D. Achter. MR2640290

[9] J. C. Lagarias, The set of primes dividing the Lucas numbers has density 2/3, Pacific J. Math. 118 (1985), no. 2, 449-461. MR789184

[10] J. C. Lagarias, Errata to: "The set of primes dividing the Lucas numbers has density $2 / 3$ " [Pacific J. Math. 118 (1985), no. 2, 449-461; MR0789184 (86i:11007)], Pacific J. Math. 162 (1994), no. 2, 393-396. MR.1251907

[11] Richard Pink, On the order of the reduction of a point on an abelian variety, Math. Ann. 330 (2004), no. 2, 275-291, DOI 10.1007/s00208-004-0548-8. MR2089426 
[12] Jeremy Rouse and David Zureick-Brown, Elliptic curves over $\mathbb{Q}$ and 2-adic images of Galois, Res. Number Theory 1 (2015), Art. 12, 34, DOI 10.1007/s40993-015-0013-7. MR3500996

[13] Joseph H. Silverman, The arithmetic of elliptic curves, Graduate Texts in Mathematics, vol. 106, Springer-Verlag, New York, 1992. Corrected reprint of the 1986 original. MR.1329092

[14] Joseph H. Silverman and John Tate, Rational points on elliptic curves, Undergraduate Texts in Mathematics, Springer-Verlag, New York, 1992. MR1171452

[15] David E. Speyer, Perfect matchings and the octahedron recurrence, J. Algebraic Combin. 25 (2007), no. 3, 309-348, DOI 10.1007/s10801-006-0039-y. MR.2317336

Department of Mathematics and Statistics, Wake Forest University, Winston-Salem, North Carolina 27109

Current address: Department of Statistics, University of Florida, Gainesville, Florida 32611

E-mail address: davibf11@ufl.edu

Department of Mathematics and Statistics, Wake Forest University, Winston-Salem, North CARolina 27109

E-mail address: rkotsonis@uchicago.edu

Department of Mathematics and Statistics, Wake Forest University, Winston-Salem, North Carolina 27109

E-mail address: rouseja@wfu.edu 